\title{
Passenger lymphocyte syndrome presented as hemolytic anemia after small bowel transplantation: a case report
}

\author{
Mihyeong Kim , Dongjin Kim², Jihyang Lim³ ${ }^{3}$ Jaehui Jung ${ }^{4}$, Jil Kim ${ }^{5}$, Jeongkye Hwang ${ }^{6}$ \\ 1Department of Surgery-Transplantation, St. Paul's Hospital, The Catholic University of Korea, Suwon, Korea
${ }^{2}$ Department of Surgery, Eunpyeong St. Mary's Hospital, The Catholic University of Korea, Seoul, Korea
${ }^{3}$ Department of Laboratory Medicine, Eunpyeong St. Mary's Hospital, The Catholic University of Korea, Seoul, Korea
${ }^{4}$ Department of Surgery-Pediatric, Seoul St. Mary's Hospital, The Catholic University of Korea, Seoul, Korea
${ }^{5}$ Department of Surgery-Transplantation, Uijeongbu St. Mary's Hospital, The Catholic University of Korea, Uijeongbu, Korea
${ }^{6}$ Department of Surgery-Transplantation, Eunpyeong St. Mary's Hospital, The Catholic University of Korea, Seoul, Korea
}

Background: The passenger lymphocyte syndrome (PLS) induces hemolytic anemia after minor ABO mismatched organ transplantation. We experienced a case of PLS with hemolysis after small bowel transplantation.

Case report: A 65-year-old male underwent massive small bowel resection and right colectomy for superior mesenteric artery embolism in January 2021. Around $30 \mathrm{~cm}$ jejunum was left and he got end jejunostomy and was completely dependent on parenteral nutrition. He received small bowel from deceased donor on June 2021. His blood type was B Rh+ and donor was $\mathrm{O} R \mathrm{R}+$. We used Simulect $20 \mathrm{mg}$, anti-thymocyte globulin (ATG) $1.5 \mathrm{mg} / \mathrm{kg}$ for 5 times and prednisolone 1,000 mg as an induction and then tacrolimus with trough level $13-15 \mathrm{ng} / \mathrm{mL}$ and reduced dose of prednisolone as a maintenance. Patient was stable and transfusion was not needed during the surgery. The hemoglobin level was decreased gradually from $10.4 \mathrm{mg} / \mathrm{dL}$ preoperatively to $5.6 \mathrm{mg} / \mathrm{dL}$ at POD \#5. There wasn't any bleeding sign on physical exam and CT angiography. A platelet count was decreased together, $60 \mathrm{~K}$ at POD \#5. We thought it might be ATG related bone marrow suppression and gave him RBCs and platelets with supportive care. The platelet count was low but sustained greater than $60 \mathrm{~K}$ and recovered over $100 \mathrm{~K}$ at POD \#23 and last transfusion was POD \#9. However, hemoglobin drop was repeated and transfusion was needed weekly till POD \#27. In isoagglutinin test, anti-B IgG was detected at 1:8 titer. A haptoglobin was lower than $20 \mathrm{mg} / \mathrm{dL}$ and direct antibody test was positive for IgG and C3bd. We already used rituximab at POD \#13 due to de novo antibody and CD19 and 20 was completely suppressed. At POD \#36, there's no edema or ulcer and normal villi was observed on endoscopy. He is stable and on a diet program to reduce parenteral nutrition.

Conclusions: Most of PLS is self-limiting but often poor outcomes have been reported, awareness and suspicion are important.

Corresponding author: Jeongkye Hwang

E-mail: jjungyong@catholic.ac.kr

(c) The Korean Society for Transplantation

This is an Open Access article distributed under the terms of the Creative Commons Attribution Non-Commercial License (http://creativecommons.org/licenses/by-nc/4.0/) which permits unrestricted non-commercial use, distribution, and reproduction in any medium, provided the original work is properly cited. 